\title{
AGENTE COMUNITÁRIO DE SAÚDE NO ESPÍRITO SANTO: DO PERFIL ÀS ATIVIDADES DESENVOLVIDAS
}

\author{
COMMUNITY HEALTH AGENT: FROM THE PROFILE TO ACTIVITIES DONE
}

AGENTE COMUNITARIO DE SALUD:DEL PERFIL A LAS ACTIVIDADES DESARROLLADAS

\author{
Ana Claudia Pinheiro Garcia ${ }^{1}$ \\ Rita de Cássia Duarte Lima ${ }^{2}$ \\ Heletícia Scabelo Galavote ${ }^{3}$ \\ Ana Paula Santana Coelho ${ }^{4}$
}

Resumo Ao considerar a amplitude e a complexidade do papel que os agentes comunitários de saúde assumem na Estratégia Saúde da Família, constituiu-se objeto do estudo apresentado neste artigo conhecer o perfil e a realidade de trabalho desses profissionais, no sentido de contribuir para a consolidação do Sistema Único de Saúde. Tratou-se de estudo quantitativo, realizado em dez municípios com população superior a 50 mil habitantes no Espírito Santo, de julho de 2012 a agosto de 2013. Foram selecionadas unidades de saúde da família com equipes completas, totalizando 121 agentes comunitários de saúde participantes do estudo. Os dados foram coletados mediante questionário estruturado autoaplicável. Os resultados revelaram que as atividades realizadas com maior frequência pelos agentes eram: visita domiciliar, atualização de cadastro, reunião de equipe e acompanhamento dos grupos prioritários definidos pelo Ministério da Saúde. Embora grande parte dos agentes fizesse o mapa inteligente e o diagnóstico de saúde, somente 13,2\% identificaram famílias de risco e $14,9 \%$ realizaram o levantamento de problemas de saúde de sua microárea. Assim, questionou-se a verdadeira finalidade do mapa inteligente e do diagnóstico de saúde, ou a forma de participação do agente na elaboração desses instrumentos, o que poderia estar restrito somente à formalização da prática. Palavras-chave atenção básica; Estratégia Saúde da Família; agente comunitário de saúde.

\author{
Elza Cléa Lopes Vieira ${ }^{5}$ \\ Renata Cristina Silva ${ }^{6}$ \\ Maria Angélica Carvalho Andrade ${ }^{7}$
}

Abstract Considering the breadth and complexity of the role that community health agents play in the Family Health Strategy, the purpose of the study presented in this article was to get to know the profile and the reality of these professionals' work to contribute to the consolidation of the Unified Health System. This was a quantitative study conducted in ten municipalities with more than 50,000 inhabitants in the state of Espírito Santo, Brazil, from July 2012 to August 2013. Family health units with complete teams were selected, and a total of 121 community health agents took part in the study. Data were collected through a self-administered structured questionnaire. The results showed that the agents' most frequent activities were home visits, registration updates, team meetings, and follow-up on the priority groups defined by the Ministry of Health. Although many of the agents completed the smart map and the health diagnosis, only 13.2 percent of them identified families at risk, and 14.9 percent surveyed the health issues of their micro-area. Thus, the true purpose of the smart map and of the health diagnosis, or of the agent's participation in the preparation of these instruments was questioned, which could be restricted only to formalize the practice.

Keywords primary care; Family Health Strategy; community health agent. 


\section{Introdução}

A Estratégia Saúde da Família (ESF) surgiu no cenário brasileiro como uma proposta de reorientação do modelo assistencial a partir da atenção básica, tendo como alicerce os princípios do Sistema Único de Saúde (SUS). Para superar o modelo assistencial hospitalocêntrico centrado no cuidado médico individualizado, visando ao reforço à participação da comunidade e ao vínculo de responsabilidade entre os serviços de saúde e a população, os serviços passaram a buscar estratégias para desenvolver uma atenção integral à saúde de indivíduos e grupos; intervir sobre fatores de risco aos quais a população está exposta; promover parcerias por meio de ações intersetoriais; e estimular o controle social (Santos et al., 2011).

Nesse contexto de implantação de políticas voltadas para a reorientação do modelo de atenção à saúde, o agente comunitário de saúde (ACS) é um profissional que merece destaque. Além da ESF, o ACS integra as equipes do Programa de Agentes Comunitários de Saúde (Pacs), realizando atividades de prevenção de doenças e promoção da saúde, por meio de ações educativas nos domicílios e na coletividade, em consonância com as diretrizes do SUS. Com o importante papel desempenhado pelo ACS, amplia-se o acesso às ações e serviços de informação e promoção social e de proteção da cidadania (Silva e Rodrigues, 2000).

Na atenção básica em saúde, o ACS é um elemento-chave do sistema, atuando como o elo entre a comunidade e os serviços. É ele quem está presente no cotidiano dos lares, quem vivencia os problemas específicos de saúde e os sociais. Também é ele quem acompanha a miséria humana em sua face mais cruel: a do abandono na doença, da falta de acesso aos serviços, da fome que mata ou debilita. E ainda é aquele que tem o privilégio de chegar primeiro aos dados, de observar as mudanças ocorridas a partir da intervenção das ações voltadas à obtenção da saúde, diretas ou não (Silva e Rodrigues, 2000).

A profissão de ACS, regulamentada na lei n. 10.507/2002, caracteriza-se pelo exercício de atividade de prevenção de doenças e promoção da saúde mediante ações domiciliares ou comunitárias, individuais ou coletivas, desenvolvidas em conformidade com as diretrizes do SUS e sob supervisão do gestor local. Para o exercício da profissão, o ACS deverá preencher os seguintes requisitos: residir na área da comunidade em que atuar; haver concluído com aproveitamento curso de qualificação básica para a formação de ACS e o ensino fundamental completo. E ainda deverá ser responsável por no máximo 750 pessoas. 
No contexto da atenção básica, esses profissionais têm atribuições definidas na Política Nacional de Atenção Básica, dentre elas: desenvolver ações que busquem a integração entre a equipe de saúde e a população adscrita à unidade básica de saúde (UBS), identificando as características e as finalidades do trabalho de acompanhamento de indivíduos e grupos sociais; trabalhar com adscrição de famílias em base geográfica definida; desenvolver ações educativas, visando à promoção da saúde e à prevenção das doenças, de acordo com o planejamento da equipe; cadastrar todas as pessoas de sua microárea; orientar as famílias quanto à utilização dos serviços de saúde disponíveis; desenvolver atividades de promoção da saúde, prevenção de doenças e agravos e de vigilância à saúde, por meio de visitas domiciliares e de ações individuais e coletivas; acompanhar, por visita domiciliar, todas as famílias e indivíduos sob sua responsabilidade, de acordo com as necessidades definidas pela equipe; e cumprir com as atribuições definidas para os ACSs em relação à prevenção e ao controle de malária e dengue (Brasil, 2011).

Ao partir da premissa de que esses profissionais devem estar comprometidos com sua realidade local e capacitados para contribuírem na minimização dos fatores que respondem pelos indicadores sociossanitários, favorecendo o reconhecimento das condições de vida da população, das suas necessidades e prioridades, a proposta da pesquisa que deu origem a este artigo partiu do entendimento de que seu perfil e suas atividades realizadas, em especial no que tange à relação com a comunidade, precisam ser mais bem definidos e especificados.

Considerando-se a amplitude e a complexidade do papel que os ACSs assumem na equipe, constituiu-se objeto do estudo aqui apresentado descrever o perfil e as atividades de trabalho desses profissionais, bem como conhecer a relação que estabelecem com a comunidade, no sentido de contribuir para a consolidação do SUS.

\section{Desenvolvimento do estudo e método}

O estudo que originou este artigo foi do tipo descritivo, transversal, de abordagem quantitativa, realizado no estado do Espírito Santo (Garcia et al., 2014). A população foi constituída por 121 ACSs que faziam parte de equipes da ESF em que os profissionais atuavam juntos há pelo menos seis meses, em municípios com mais de 50 mil habitantes. Um dos municípios foi excluído por não apresentar nenhuma equipe que atendesse a esse critério. Não foram considerados aqueles que atuavam no Pacs (Quadro 1). 
Quadro 1

\begin{tabular}{|ccccc}
\hline $\begin{array}{c}\text { Municípios capixabas com mais de } 50 \text { mil habitantes: população, número de equipes de saúde } \\
\text { da família, cobertura da ESF e número de ACSs atuantes no Pacs e na ESF, 2012 }\end{array}$ \\
\hline Período & População1 & Equipes de SF22 & Cobertura da ESF3 & ACSs3 $^{3}$ \\
\hline Aracruz & 81.832 & 23 & $74,68 \%$ & 145 \\
Cachoeiro de Itapemirim & 189.889 & 29 & $54,18 \%$ & 228 \\
Cariacica & 348.738 & 23 & $11,81 \%$ & 194 \\
Colatina & 111.788 & 25 & $67,51 \%$ & 194 \\
Guarapari & 105.286 & 6 & $16,18 \%$ & 139 \\
Linhares & 141.306 & 24 & $64,91 \%$ & 296 \\
São Mateus & 109.028 & 17 & $40,61 \%$ & 207 \\
Serra & 409.267 & 36 & $26,54 \%$ & 245 \\
Viana & 65.001 & 8 & $47,13 \%$ & 141 \\
Vila Velha & 414.586 & 35 & $77,24 \%$ & 188 \\
Vitória & 327.801 & 77 & $77,24 \%$ & 372 \\
Total & 2.304 .522 & 303 & $49,62 \% 4$ & 2.349 \\
& & &
\end{tabular}

Fonte: 1Instituto Brasileiro de Geografia e Estatística (2010); ${ }^{2}$ As autoras; ${ }^{3 B}$ Brasil (2012).

Nota 1: SF - Saúde da Família; ESF - Estratégia Saúde da Família; ACSs - Agentes comunitários de saúde. Nota 2: 4 Média da população total dos municípios cobertos pela ESF.

Uma vez definidas as equipes participantes, foram entregues, nas próprias unidades de saúde, questionários semiestruturados e autoaplicáveis aos coordenadores das unidades ou aos enfermeiros responsáveis pelas equipes. A esses profissionais foram fornecidas orientações sobre o preenchimento do instrumento e explicados os objetivos da pesquisa e sua importância para a obtenção de avanços relacionados à saúde da família e à atenção primária à saúde no Espírito Santo, tendo sido solicitado que se repassassem tais informações aos ACSs. Posteriormente, os instrumentos foram recolhidos pelos pesquisadores.

A aplicação dos questionários ocorreu no período de julho de 2012 a agosto de 2013, com uma perda de 4,6\%, uma vez que seis questionários não foram devolvidos. A construção desses instrumentos baseou-se em pesquisa realizada anteriormente em quatro capitais brasileiras, entre elas o município de Vitória (Giovanella, Escorel e Mendonça, 2009). No Espírito Santo, realizou-se um pré-teste, no município de Domingos Martins, em 2011. Os questionários estavam estruturados ao redor de quatro tópicos: perfil do profissional, inserção e capacitação na ESF, organização do processo de trabalho na ESF e coordenação e integração na rede dos serviços de saúde. Contavam com setenta questões. Para o estudo desenvolvido, consideraram-se apenas os tópicos referentes ao perfil dos ACSs e à organização do processo de trabalho. 
Realizou-se a análise descritiva dos dados por meio do pacote estatístico SPSS 18.0. Os dados foram apresentados na forma de tabela, em frequência absoluta e relativa.

Por envolver seres humanos, a fim de atender aos requisitos estabelecidos pela resolução n. 466/2012, do Conselho Nacional de Saúde, o estudo foi encaminhado ao Comitê de Ética em Pesquisa da Universidade Federal do Espírito Santo (Ufes), tendo sido aprovado de acordo com o parecer n. 255/11. A pesquisa contou com financiamento da Fundação de Amparo à Pesquisa e Inovação do Espírito Santo (Fapes) e do Conselho Nacional de Desenvolvimento Científico e Tecnológico (CNPq).

\section{Perfil dos profissionais}

Observa-se, na Tabela 1, a prevalência de ACSs do sexo feminino. A predominância de profissionais do sexo feminino vai ao encontro da crescente feminização verificada no campo da saúde em geral. Em relação aos ACSs, confirma os resultados apontados em outros trabalhos (Galavote et al., 2011; Santos et al., 2011; Ursine, Trelhas e Nunes, 2010). Silva e Rodrigues (2010) destacam o papel cuidador comumente desempenhado pela mulher na sociedade, sobretudo em relação às crianças e aos idosos, conferindo a esses profissionais mais credibilidade e sensibilidade com a comunidade. Mota e David (2010) afirmam que a maior parte dos ACSs em todas as regiões do país é mulher, chamando a atenção para o fato de que, geralmente, profissões associadas ao papel feminino são marcadas pela precarização e instabilidade.

\section{Tabela 1}

\begin{tabular}{lcc}
$\begin{array}{l}\text { Caracterização dos ACSs das equipes de saúde da família em municípios com } \\
\text { mais de } 50 \text { mil habitantes - Espírito Santo, 2012/2013 }\end{array}$ & $\%$ \\
\hline \multicolumn{1}{c}{ Características } & N & \\
\hline Sexo & 113 & $93,4 \%$ \\
$\quad$ feminino & 8 & $6,6 \%$ \\
$\quad$ masculino & 15 & $12,6 \%$ \\
Faixa etária & 52 & $43,1 \%$ \\
menos de 30 anos & 33 & $27,4 \%$ \\
entre 30 e 39 anos & 17 & $14,2 \%$ \\
entre 40 e 49 anos & 2 & $1,6 \%$ \\
entre 50 e 59 anos & 2 & $1,6 \%$ \\
$\quad 60$ anos ou mais & & \\
não respondeu & 111 & $91,7 \%$ \\
Escolaridade & 4 & $3,3 \%$ \\
Ensino médio completo &
\end{tabular}

Fonte: Pesquisa Perfil dos Profissionais de Saúde da Estratégia Saúde da Família do Espírito Santo (Garcia et al., 2014). 
A faixa etária predominante $(43,1 \%)$ estava compreendida entre 30 e 39 anos, e o segundo maior contingente $(27,4 \%)$ tinha entre 40 e 49 anos. Segundo Ferraz e Aertz (2005), é maior a probabilidade de ACSs com mais idade terem mais conhecimento sobre os problemas da comunidade, assim como mais vínculo e laços de amizade. Contudo, mudanças fundamentais podem ser alcançadas para se consolidar a ESF e, assim, reorganizar-se o sistema de saúde no país, com a entrada de profissionais jovens.

No que diz respeito à formação dos ACSs, a escolaridade predominante foi o ensino médio completo $(91,7 \%)$. Ainda que tenha se verificado um aumento na escolaridade do brasileiro nos últimos anos, é importante salientar que, com a implantação do Programa Saúde da Família, ampliou-se o papel desses profissionais, com a exigência de novas competências no campo social e político, o que requer um grau de escolaridade mais complexo e abrangente. Marzari, Junges e Selli (2011) destacam a preocupação dos ACSs em relação ao elemento qualificação, pois reconhecem a necessidade de profissionalização como unânime no discurso desses atores. Os autores ressaltam que a profissionalização e a qualificação do ACS é um fator importante na constituição do seu trabalho cotidiano, mas alertam que a dificuldade em criar uma identidade legítima no trabalho pode levá-lo a buscar conhecimentos que se equiparam aos saberes dos outros profissionais da equipe.

A diversidade do trabalho do ACS sinaliza a necessidade de constante atualização conceitual. No entanto, a indefinição do seu papel no escopo da equipe e da comunidade pode gerar a busca equivocada de conhecimentos que não condizem com as competências requeridas pelo seu trabalho: observação, ética, noção de causa e consequência, comunicação, integração, liderança, responsabilidade e autonomia (Marzari, Junges e Selli, 2011).

Galavote e colaboradores (2011) apontam que os ACSs almejam outra inserção profissional no futuro, em especial os mais jovens, o que acarreta a busca por cursos de qualificação e profissionalização que permitam a formação para outras funções que garantam maior rentabilidade financeira.

\section{Atividades realizadas pelos ACSs}

Para a identificação das atividades realizadas com maior frequência pelos ACSs, foi elaborada uma questão com 25 opções fechadas, baseadas nas atribuições definidas pela Política Nacional de Atenção Básica (Brasil, 2011), com a possibilidade de que mais de uma fosse selecionada, e uma última opção, na qual eles poderiam especificar outra(s) atividade(s) que não estivesse(m) listada(s). A Tabela 2 apresenta a frequência das atividades referidas pelos ACSs em estudo, com destaque para visita domiciliar $(90,1 \%)$, atualização de cadastro $(51,2 \%)$ e reunião de equipe $(48,8 \%)$. Também é expressivo o 
contingente que afirma fazer acompanhamento a gestantes $(41,3 \%)$ e controle de hipertensão, diabetes, tuberculose e hanseníase $(41,3 \%)$.

\section{Tabela 2}

Atividades realizadas com maior frequência pelo ACS das equipes de saúde da família, em municípios com mais de 50 mil habitantes - ES, 2012/2013

\begin{tabular}{|c|c|c|}
\hline Atividades & $\mathrm{N}$ & $\%$ \\
\hline $\begin{array}{l}\text { Trabalho de campo desenvolvendo atividades dirigidas para } \\
\text { as famílias }\end{array}$ & 31 & $25,6 \%$ \\
\hline $\begin{array}{l}\text { Trabalho de campo desenvolvendo atividades dirigidas para } \\
\text { a comunidade em geral }\end{array}$ & 8 & $6,6 \%$ \\
\hline $\begin{array}{l}\text { Atividades de capacitação continuada sobre temas } \\
\text { relacionados às práticas de cuidados exercidas pela equipe }\end{array}$ & 6 & $5,0 \%$ \\
\hline Visita domiciliar & 109 & $90,1 \%$ \\
\hline Reunião de equipe & 59 & $48,8 \%$ \\
\hline Atualização do cadastro & 62 & $51,2 \%$ \\
\hline Identificação das famílias de risco & 16 & $13,2 \%$ \\
\hline Busca ativa de grupos prioritários & 22 & $18,2 \%$ \\
\hline Acompanhamento do crescimento e desenvolvimento infantil & 23 & $19,0 \%$ \\
\hline Acompanhamento a gestantes & 50 & $41,3 \%$ \\
\hline Prevenção de DST/Aids & 11 & $9,1 \%$ \\
\hline Prevenção da dengue & 33 & $27,3 \%$ \\
\hline Informação sobre imunização infantil & 35 & $28,9 \%$ \\
\hline Informação sobre reidratação oral (soro caseiro) & 13 & $10,7 \%$ \\
\hline Incentivo ao aleitamento materno & 33 & $27,3 \%$ \\
\hline Informação sobre planejamento familiar & 19 & $15,7 \%$ \\
\hline Informação sobre alimentação saudável & 13 & $10,7 \%$ \\
\hline Prevenção do câncer de colo de útero & 25 & $20,7 \%$ \\
\hline Controle de hipertensão, diabetes, tuberculose ou hanseníase & 50 & $41,3 \%$ \\
\hline Cuidado de idosos & 20 & $16,5 \%$ \\
\hline Acompanhamento a enfermeiros e médicos nas visitas domiciliares & 43 & $35,5 \%$ \\
\hline Agendamentos e encaminhamentos necessários & 18 & $14,9 \%$ \\
\hline $\begin{array}{l}\text { Levantamento dos problemas de saúde da população de } \\
\text { sua microárea }\end{array}$ & 18 & $14,9 \%$ \\
\hline $\begin{array}{l}\text { Preenchimento dos formulários de informações para o } \\
\text { Sistema de Informação da Atenção Básica (Siab) }\end{array}$ & 31 & $25,6 \%$ \\
\hline Reuniões com a coordenação/supervisão & 16 & $13,2 \%$ \\
\hline $\begin{array}{l}\text { Utilização de mapa inteligente da área de atuação no qual } \\
\text { estão discriminadas as microáreas de responsabilidade dos ACSs }\end{array}$ & 102 & $84,3 \%$ \\
\hline $\begin{array}{l}\text { Realização de diagnóstico da situação de saúde da população } \\
\text { identificando os problemas mais frequentes }\end{array}$ & 115 & $95,0 \%$ \\
\hline
\end{tabular}

Fonte: Pesquisa Perfil dos Profissionais de Saúde da Estratégia Saúde da Família do Espírito Santo (Garcia et al., 2014). 
A realização de visita domiciliar constitui o cerne do trabalho do ACS e é prevista como uma das suas atribuições de acordo com a Política Nacional de Atenção Básica, com base em critérios de risco e vulnerabilidade, acompanhada pelo cadastramento das famílias residentes em uma unidade territorial definida como microárea, orientação da utilização dos serviços de saúde, ações de promoção, prevenção e vigilância dos agravos e integração entre a comunidade e a equipe da ESF (Brasil, 2011). A frequência de realização das visitas domiciliares fundamenta-se na recomendação da referida política com uma visita/família/mês, e os usuários com maior vulnerabilidade serão definidos como prioridade no acompanhamento realizado pelos ACSs. Por ser uma atribuição básica no cotidiano de trabalho, foi referenciada neste estudo como a principal atividade (90,1\%); no entanto, cerca de $9 \%$ dos agentes não a citaram como uma das funções principais constituintes do seu escopo de ações. Galavote e colaboradores (2011), em estudo realizado em Vitória, no Espírito Santo, apontam que a visita domiciliar é reconhecida pelos ACSs como um requisito essencial para o desenvolvimento de seu trabalho; consideram como um espaço de encontro fecundo entre o trabalhador e o usuário por meio de relações intercessoras, um cenário de disputas, desejos, intencionalidades, pactuações e agenciamentos. Para Merhy (2002), essas relações de intercessão partilhada oriundas nesse espaço de encontro permitem que o usuário constitua parte do processo, um agente do cuidado, desde que a natureza do trabalho seja viva em ato, isto é, que permita o protagonismo do trabalhador e do usuário como atores desse processo - o que para o ACS só é possível mediante o estabelecimento de uma relação sustentada pelo vínculo e responsabilização. A visita domiciliar pode ainda significar uma intromissão no âmbito privado tido como o domicílio do usuário, o que constitui, frequentemente, um dificultador no estabelecimento do vínculo (Franco e Merhy, 1999).

A constatação de que $9 \%$ dos ACSs em estudo não referenciavam a visita domiciliar como uma das atividades mais frequentes do seu cotidiano alertou para o sentido real dessa atividade na ESF. Albuquerque e Bosi (2009) reconhecem a visita domiciliar como um espaço de encontro entre o trabalhador e o usuário, no qual é possível o estabelecimento de vínculo e responsabilização por meio da interface de diálogo entre os atores na produção dos atos em saúde. No entanto, os aspectos subjetivos oriundos da execução da visita domiciliar podem sinalizar a instância sofrimento para o ACS, expressa no discurso da limitação de atuação e resolutividade perante as demandas da população e nas falhas de comunicação entre o trabalhador e a comunidade.

A credibilidade do agente perante a comunidade constitui um fator determinante do sucesso da interação no momento da visita domiciliar e é determinada pela resolução das demandas da população, as quais na maioria das vezes se referem a aspectos da organização do serviço e da rede de 
atenção à saúde, o que gera a fragilização dessa atividade no cotidiano desse trabalhador (Jardim e Lancman, 2009). Assim, a visita domiciliar pode representar o cerne do trabalho do ACS como espaço de constituição do seu saber-fazer no encontro com o usuário ou, então, ser designada como um momento de intenso sofrimento com ruídos e falhas na construção da relação trabalhador-comunidade.

As atividades de campo direcionadas à comunidade foram citadas por $6,6 \%$ dos ACSs, considerando que outras atividades referenciadas contemplavam esse domínio. O trabalho do ACS no espaço extramuros representa a essência do fazer, já que sua atuação prevê a proposição de ações de saúde in loco, ou seja: a proximidade com o espaço de vida do usuário constitui a possibilidade de identificação de vulnerabilidades que orientam o projeto terapêutico singular desenvolvido pela equipe de saúde.

Menos de $50 \%$ dos entrevistados apontaram a reunião de equipe como uma das atividades mais frequentes no cotidiano. A reunião de equipe pode representar um momento fecundo de construção de saberes e reflexão das práticas com base na vivência coletiva no trabalho ou ser uma atividade meramente burocrática da execução de ações de cunho administrativo, como o fechamento da produção mensal da unidade de saúde, sendo relegada a uma atribuição da organização do serviço e não uma construção do cotidiano do trabalho em saúde.

Grando e Dall' Agnol (2010) identificam dificuldades do processo grupal em reuniões de equipes na ESF, com observância do desenvolvimento de uma prática protocolar, meramente técnica, em detrimento de estruturação, organização e tomada de decisões no espaço de constituição do trabalho nesse cenário. As autoras evidenciam momentos de exclusão da opinião e percepção dos membros da equipe com a transmissão de informações que confere à reunião um caráter apenas informativo.

A reunião de equipe no escopo de ações de uma unidade de saúde da família representa a possibilidade de encontro entre os trabalhadores, um espaço propício à proposição de novos conhecimentos se for vinculada a sua legitimidade como compartilhamento de saberes e valorização dos discursos dos atores, independentemente do papel que ocupam no serviço. Menegussi, Ogata e Rosalini (2014) alertam sobre o silenciamento do ACS perante a equipe, pois não encontra espaço para a proposição de suas percepções oriundas do trabalho em sua microárea.

Galavote e colaboradores (2011) referem que o ACS expressa uma pormenorização do seu saber perante os saberes instituídos da equipe, estando à margem dos processos decisórios e reconhecendo a limitação do seu saber-fazer em relação à diversidade com que se depara no trabalho. O que existe é uma desvalorização coletiva dos diferentes saberes perante o saber biomédico legitimado. Em estudo realizado por Ferreira e colaboradores (2009), observa-se 
que a existência de poucos espaços de escuta e diálogo na equipe não tem permitido a participação do ACS nos processos decisórios relacionados à gestão do cuidado, à organização e ao funcionamento dos serviços de saúde.

A atualização do cadastro corresponde à vertente técnica do trabalho do ACS que pode se sobrepor à vertente assistencial, por meio da valorização das atividades de cunho administrativo em superioridade às ações de atenção direta à comunidade. Nogueira, Silva e Ramos (2000) afirmam que tal aspecto constitui um dilema permanente na prática do agente, que se vê 'dividido' entre a dimensão técnica e a social de seu trabalho, o que gera conflitos evidentes na prática cotidiana. No estudo apresentado neste artigo, a atualização do cadastro $(51,2 \%)$ apareceu como a segunda atividade mais frequente no trabalho do ACS, sendo mais citada do que as atividades assistenciais, especialmente aquelas direcionadas aos grupos prioritários definidos pela avaliação das reais necessidades de saúde dos usuários.

O campo de conhecimentos do ACS se constrói com base nos saberes que ele adquire no convívio com os profissionais da equipe; nas capacitações de que participa em temas afins como hanseníase, tuberculose, saúde da criança e saúde da mulher, entre outros; e no conhecimento que traz consigo das experiências de vida relacionadas à sua subjetividade - que para Deleuze (2001) representa uma dupla potência, à medida que crê e inventa, presume poderes secretos e supõe poderes abstratos, distintos. No estudo aqui apresentado, apenas $5 \%$ dos ACSs referiram desenvolver com frequência atividades de educação continuada com temas relacionados ao seu cotidiano de trabalho, o que gerava uma limitação do seu saber-fazer em face da complexidade com que se deparavam no trabalho no espaço intra e extramuros.

No que tange à utilização de mapa inteligente da área de atuação, 84,3\% dos entrevistados afirmaram utilizá-lo e 95,5\% relataram realizar diagnóstico de situação de saúde da população de seu território, sendo essas as atividades que mais realizavam. A identificação dos problemas de saúde do território é prática fundamental para a reorganização das práticas em saúde com foco nas necessidades de saúde da população, assim como para o alcance da integralidade da atenção. Segundo a Política Nacional de Atenção Básica, faz parte do processo de trabalho das equipes de atenção básica desenvolver a programação e implementação das atividades de atenção à saúde de acordo com os problemas e necessidades da população, com a priorização de intervenções clínicas e sanitárias nos problemas de saúde segundo critérios de frequência, risco, vulnerabilidade e resiliência (Brasil, 2011).

Para Silva, Batistella e Gomes (2007), a incorporação da noção de problema de saúde no processo de planejamento e programação das ações na atenção básica possibilita a valorização de uma visão mais policêntrica e democrática na definição de seus objetos de intervenção. Em perspectiva mais integral, a noção de problema de saúde inclui, além da doença, dos 
modos de transmissão e dos fatores de risco, as necessidades e/ou determinantes dos modos de vida e saúde (condições de vida e trabalho), identificados não somente pelos enfoques clínico e epidemiológico, mas sobretudo pelo enfoque social.

O mapa inteligente é uma ferramenta da territorialização que permite analisar no espaço as informações a respeito das condições de vida e saúde da população adscrita; é, portanto, uma forma de diagnóstico do território. Para Monken e Barcellos (2007), a análise territorial implica uma coleta sistemática de dados que vão informar sobre situações-problema naquela população e naquele território, indicando suas inter-relações espaciais. Possibilita, ainda, identificar vulnerabilidades, populações expostas e a seleção de problemas prioritários para as intervenções - o que permite a escolha de ações mais adequadas, constituindo-se em ferramenta poderosa para o planejamento por intermédio da microlocalização dos problemas de saúde.

Por meio dos dados coletados, verificou-se uma contradição. Embora grande parte dos ACSs tenha afirmado realizar com frequência mapa inteligente e diagnóstico de saúde, somente 13,2\% apontaram a identificação de famílias de risco - e 14,9\% o levantamento de problemas de saúde de sua microárea - como ações prioritárias. Dessa forma, questionou-se a verdadeira finalidade do mapa inteligente e do diagnóstico de saúde, ou o entendimento desses atores em relação a essas ferramentas. Deve-se refletir ainda sobre a forma de participação do ACS na elaboração desses instrumentos, que pode estar restrito somente à formalização da prática, e não a uma utilização efetiva e consciente com base em sua devida finalidade. Essa constatação pode refletir a posição desse trabalhador à margem dos processos decisórios da equipe, não encontrando espaço no bojo da organização e no planejamento das ações, uma vez que o diagnóstico da situação de saúde se constitui no primeiro momento do processo de planejamento.

\section{Relação entre os ACSs e a comunidade}

Para Nogueira, Silva e Ramos (2000), o ACS pode ser visto como um trabalhador 'genérico', pois não pertence a nenhuma categoria profissional da área da saúde e realiza atividades que ultrapassam esse campo. Desempenha importante papel social como mediador entre as distintas esferas de organização da vida social e identidade comunitária, que o diferencia dos demais trabalhadores da saúde. O ACS é referenciado como o elo entre a equipe de saúde e a comunidade, e considerado o principal responsável pela efetivação do vínculo nessa relação.

A pesquisa aqui apresentada encontrou resultados satisfatórios no tocante à relação entre os ACSs e a comunidade. Na Tabela 3, observa-se que 
$51,2 \%$ dos agentes afirmaram que costumam procurar a família do usuário para discutir os problemas de saúde, o que ocorria na maioria das vezes, enquanto $31,4 \%$ declararam que o faziam sempre.

Tabela 3

Relação entre ACSs das equipes de saúde da família e a comunidade, em municípios com mais de 50 mil habitantes - Espírito Santo, 2012/2013

\begin{tabular}{lcc}
\hline \multicolumn{1}{c}{ Relação entre profissionais e comunidade } & N & \\
\hline Se costuma procurar a família do usuário para discutir & & \\
os problemas de saúde & 38 & $31,4 \%$ \\
$\quad$ Sempre & 62 & $51,2 \%$ \\
$\quad$ Na maioria das vezes & 18 & $14,9 \%$ \\
$\quad$ Poucas vezes & 3 & $2,5 \%$ \\
$\quad$ Nunca & & \\
Se durante a realização de procedimentos/atividades & & \\
de saúde pergunta sobre fatores de risco social ou & & \\
condições de vida do usuário & 73 & $60,3 \%$ \\
$\quad$ Sempre & 36 & $29,8 \%$ \\
$\quad$ Na maioria das vezes & 10 & $8,3 \%$ \\
Poucas vezes & 1 & $0,8 \%$ \\
$\quad$ Nunca & & \\
Se desenvolve ações permanentes de esclarecimento à & & $68,6 \%$ \\
população sobre as características da ESF & 83 & $28,9 \%$ \\
Sim & 35 & \\
Não & & \\
\hline
\end{tabular}

Fonte: Pesquisa Perfil dos Profissionais de Saúde da Estratégia Saúde da Família do Espírito Santo (Garcia et al., 2014).

Segundo o Ministério da Saúde, as atribuições do ACS estão relacionadas a um aspecto estruturante: o papel de educador, no intuito de gerar mudança na comunidade (Brasil, 2011). O ACS, ao procurar as famílias para discutir os problemas de saúde, atua nessa concepção de educador. Uma questão a ser considerada é a necessidade da qualificação técnica desses trabalhadores, visto esta ser desestruturada e insuficiente para subsidiar seu amplo papel na ESF.

Marzari, Junges e Selli (2011), ao analisarem a formação e o perfil profissional dos ACSs, atribuem dois perfis de agentes com seu respectivo processo de formação: o primeiro é o de promotor de conscientização, mobilização e organização social da comunidade; e o segundo refere-se a uma ação mais sanitária com formação técnica em harmonia com as outras categorias profissionais. Nesse contexto, os autores ressaltam a importância da qualificação técnica desses trabalhadores, mas alertam que ela deve estar pautada na realidade de cada comunidade e envolver toda a equipe na busca 
do conhecimento coletivo dos riscos e vulnerabilidades de cada contexto e na prática identitária de cada ACS. Na verdade, o trabalho cotidiano exige constante atualização, mas os cursos e formações propostos pelas secretarias de saúde não podem ser operados de forma isolada e descontextualizada.

Verifica-se também na Tabela 3 que 60,3\% dos ACSs sempre perguntavam sobre fatores de risco social ou condições de vida do usuário, e 29,8\% o faziam na maioria das vezes. O ACS, além de trabalhador, é sujeito da comunidade onde trabalha, por isso pode encontrar barreiras para criar vínculo e ter a confiança dos usuários. Lunardelo (2004) afirma que o domicílio não é um território público e o acesso a ele nem sempre é fácil; portanto, para realizar seu trabalho, o agente deve estabelecer vínculo e relação de confiança com a comunidade. $\mathrm{O}$ agente somente conhece a intimidade dos usuários com permissão da família e deve sempre preservar a privacidade desses usuários.

Jardim e Lancman (2009), ao analisarem os aspectos subjetivos do trabalho do ACS referentes ao morar e trabalhar na mesma comunidade, ressaltam que esses trabalhadores vivenciam constrangimentos decorrentes do fato de pertencerem à mesma comunidade na qual desempenham seu papel profissional, já que se deparam com aspectos da privacidade dos usuários, violência e assédio em relação ao seu papel de agente comunitário nos diferentes espaços públicos. O pertencer à mesma comunidade pode representar um facilitador no estabelecimento do vínculo com o usuário, mas ao mesmo tempo expõe o trabalhador em suas limitações no campo de atuação, que na maioria das vezes transpõem sua governabilidade.

Ações permanentes de esclarecimento à população sobre as características da ESF eram desenvolvidas segundo 68,6\% dos respondentes. Documentos oficiais enfatizam a importância da ESF na reorientação do modelo de atenção a partir da atenção básica, assim como consideram que esta estratégia pode impulsionar nova dinâmica na organização dos serviços e ações de saúde. Esse modelo procura agir sobre as necessidades em saúde, com orientação para a prevenção em vez da cura de doenças, rompendo com a visão médico-centrada (Bornstein e Stotz, 2008).

Bornstein e Stotz (2008) ressaltam que o agente comunitário é o trabalhador que tem maior conhecimento sobre a dinâmica social, os valores, as formas de organização e o conhecimento que circula entre os moradores de sua comunidade. Assim, é imprescindível que esse profissional exerça o papel de ouvinte da comunidade, mas também o de porta-voz da equipe de saúde, uma vez que esse conhecimento pode facilitar o trânsito da equipe, as parcerias e articulações. Porém, tais características geram diferentes expectativas pelos outros profissionais da equipe de saúde e pelos moradores. De um lado, pela inserção no serviço, espera-se que ele tenha controle da situação de saúde da população; de outro, os moradores esperam que o agente facilite seu acesso ao serviço de saúde. 
O papel de integração da comunidade com a equipe da ESF proposto ao ACS traz demandas e necessidades de saúde que não dependem sumariamente da atuação do agente, já que estão relacionadas, frequentemente, a aspectos da organização dos serviços de saúde, como a marcação de consultas e exames. O ACS não é um gerente solitário das redes de atenção à saúde, e a baixa resolutividade das demandas decorrentes do trabalho no espaço da comunidade gera a vivência de impotência, aliada à exposição excessiva dos ACSs expressa em desqualificação no trabalho, por meio da falta de reconhecimento que leva ao enfraquecimento identitário e consequentemente à perda do sentido do trabalho (Jardim e Lancman, 2009).

Galavote e colaboradores (2011) referem que o reconhecimento do trabalho representa a principal instância capaz de gerar prazer no cotidiano dos ACSs, por ser capaz de aumentar a sua potência para agir no encontro com o usuário. Segundo os autores, a motivação como desejo de permanecer na profissão é expressa na possibilidade de ajudar o outro, na construção de vínculos de amizade e no reconhecimento de que o trabalho realizado é capaz de disparar processos de autocuidado e ressignificação por parte dos usuários, que se empoderam do seu próprio cuidado e reconhecem no ACS um agente de transformação e resolutividade.

\section{Considerações finais}

A análise do perfil dos ACSs da ESF no estado do Espírito Santo revelou um trabalhador híbrido que fundamenta o seu trabalho nas atribuições definidas pela Política Nacional de Atenção Básica, com predomínio das atividades de visita domiciliar, reunião de equipe, atualização dos cadastros e formulários institucionais e acompanhamento dos grupos de risco definidos pelo Ministério da Saúde.

A reunião de equipe foi referenciada por menos de $50 \%$ dos agentes comunitários em estudo e constituiu um momento importante de reflexão de práticas e integração entre os trabalhadores da equipe, sendo um espaço de definição de elementos delineadores do planejamento e avaliação em saúde. No entanto, pode representar para os trabalhadores uma obrigatoriedade da organização do serviço com um objetivo informativo e burocrático, e não ser compreendida como um facilitador do trabalho como cenário de diálogo e valorização dos diferentes saberes.

O trabalho no espaço da comunidade configurou a essência do trabalho do ACS, o que possibilitou a proximidade e a vinculação necessária para a identificação das necessidades de saúde de cada usuário. No estudo aqui apresentado, a visita domiciliar surgiu como a atividade de maior frequência desenvolvida nesse cenário, o que sugeriu a compreensão do seu sentido real 
como prática promotora de vínculo e responsabilização. No entanto, pode gerar a instância sofrimento quando há rupturas no diálogo do ACS com a comunidade, e falhas nos processos mútuos de reconhecimento no trabalho.

Constituiu um dispositivo analisador que emergiu no estudo aqui apresentado a definição de que a maioria dos ACSs referiu como atividades mais realizadas a utilização do mapa inteligente e a construção do diagnóstico situacional do cenário de trabalho, em contradição a 14,9\% que tinham entre as suas principais ações o levantamento de problemas de saúde de sua microárea. Tal fato suscitou dúvidas sobre esses instrumentos de planejamento das ações com base na definição do critério de risco estarem sendo construídos com base naqueles nós críticos identificados pelos ACSs no cotidiano de trabalho. Essa constatação poderia refletir ainda a posição desse trabalhador à margem dos processos decisórios da equipe, não encontrando espaço no bojo da organização para a pactuação de prioridades definidas com base na análise da situação de saúde e identificação de vulnerabilidades que orientam o planejamento das ações na ESF, com base no seu olhar aproximado do espaço familiar extramuros.

Assim, a produção de saberes e as práticas dos ACSs devem propiciar infindáveis possibilidades da relação com o outro e com eles mesmos, de forma a potencializar uma produção de saúde vinculada à cidadania, à autonomia dos sujeitos e coletividades nos modos como, no dia a dia, vão se construindo novas formas de viver e lidar com a vida, inclusive nos espaços institucionais onde se constroem as diferenciadas modelagens do trabalho em saúde.

\section{Colaboradoras}

Ana Claudia Pinheiro Garcia e Rita de Cássia Duarte Lima responsabilizaram-se pela concepção do projeto, análise e interpretação dos dados, redação do artigo, revisão crítica e aprovação final da redação. Heletícia Galavote, Ana Paula Santana Coelho e Elza Cléa responsabilizaram-se pela análise e interpretação dos dados, redação do artigo, revisão crítica e aprovação final da redação. Renata Cristina Silva participou da coleta, tabulação de dados e aprovação final da redação. Maria Angélica Carvalho Andrade responsabilizou-se pela análise e interpretação dos dados, redação do artigo, revisão crítica e aprovação final da redação. 
Resumen Al considerar la amplitud y la complejidad del papel que los agentes comunitarios de salud asumen en la Estrategia Salud de la Familia, se constituyó en objeto de estudio presentado en este artículo, conocer el perfil y la realidad de trabajo de estos profesionales, desde el punto de vista de su contribución para consolidad el Sistema Único de Salud. Se trató de un estudio cuantitativo, realizado en diez municipios con población superior a 50 mil habitantes, en el estado de Espírito Santo, Brasil, de julio de 2012 a agosto de 2013. Se seleccionaron unidades de salud de la familia con equipos completos, totalizando 121 agentes comunitarios de salud participantes del estudio. Los datos se recolectaron mediante un cuestionario estructurado autoadministrado. Los resultados revelaron que las actividades realizadas con mayor frecuencia por los agentes eran: visita domiciliaria, actualización de registro, reunión de equipo y seguimiento de los grupos prioritarios definidos por el Ministerio de la Salud. Aunque gran parte de los agentes hizo el mapa inteligente y el diagnóstico de salud, sólo el 13,2\% identificaron familias de riesgo y el 14.9\% realizaron el relevamiento de problemas de salud de su microárea. Así, se cuestionó la verdadera finalidad del mapa inteligente y del diagnóstico de salud, o la forma de participación del agente en la preparación de estos instrumentos, que podría restringirse solamente a la formalización de la práctica.

Palabras clave atención básica; Estrategia Salud de la Familia; agente comunitario de salud.

\section{Notas}

1 Universidade Federal do Espírito Santo, Programa de Pós-Graduação em Saúde Coletiva, Vitória, Espírito Santo, Brasil.

<anacpgarcia@hotmail.com>

Correspondência: Rua Hélio Marconi, 170, apto. 403, Bento Ferreira, CEP 29050-690, Vitória, Espírito Santo, Brasil.

2 Universidade Federal do Espírito Santo, Programa de Pós-Graduação em Saúde Coletiva, Vitória, Espírito Santo, Brasil.

$<$ ritacdl@uol.com.br>

3 Universidade Federal do Espírito Santo, Centro Universitário Norte do Espírito Santo, Vitória, Espírito Santo, Brasil.

$<$ heleticiagalavote@yahoo.com.br>

4 Universidade Federal do Espírito Santo, Centro Universitário Norte do Espírito Santo, Vitória, Espírito Santo, Brasil.

<apscoelho@gmail.com>

5 Universidade Federal do Espírito Santo, Vitória, Espírito Santo, Brasil.

<elzaclea@gmail.com>

6 Universidade Federal do Espírito Santo, Vitória, Espírito Santo, Brasil.

$<$ renata.cristinasilva@hotmail.com>

7 Universidade Federal do Espírito Santo, Programa de Pós-Graduação em Saúde Coletiva, Vitória, Espírito Santo, Brasil.

<geliandrade@hotmail.com> 


\section{Referências}

ALBUQUERQUE, Adriana. B. B.; BOSI, Maria Lucia. M. Visita domiciliar no âmbito da Estratégia Saúde da Família: percepções de usuários no município de Fortaleza, Ceará, Brasil. Cadernos de Saúde Pública, Rio de Janeiro, v. 25, n. 5, p. 1.103-1.112, 2009.

BORNSTEIN, Vera J.; STOTZ, Eduardo N. O trabalho dos agentes comunitários de saúde: entre a mediação convencedora e a transformadora. Trabalho, Educação e Saúde, Rio de Janeiro, v. 6, n. 3, p. 457-480, nov. 2008-fev. 2009.

BRASIL. Ministério da Saúde. Portaria n. 2.488, de 21 de outubro de 2011. Aprova a Política Nacional de Atenção Básica, estabelecendo a revisão de diretrizes e normas para a organização da atenção básica, para a Estratégia Saúde da Família-ESF e o Programa de Agentes Comunitários de Saúde-Pacs. Diário Oficial [da] República Federativa do Brasil, Brasília, DF, 24 out. 2011. Disponível em: <http://bvsms.saude.gov.br/bvs/saudelegis/ gm/2011/prt2488_21_10_2011.html >. Acesso em: 18 out. 2016 .

BRASIL. Ministério da Saúde. Sala de apoio à gestão estratégica. 2012. Disponível em: < http:// sage.saude.gov.br>. Acesso em: 18 out. 2016.

DELEUZE, Gilles. Empirismo e subjetividade: ensaio sobre a natureza humana segundo Hume. São Paulo: Editora 34, 2001.

FERRAZ, Lucimare; AERTS, Denise R. G. C. O cotidiano de trabalho do agente comunitário de saúde no PSF em Porto Alegre. Ciência \& Saúde Coletiva, Rio de Janeiro, v. 10, n. 2, p. 347-355, abr.-jun. 2005.

FERREIRA, Vitória S. C. et al. Processo de trabalho do agente comunitário de saúde e a reestruturação produtiva. Cadernos de Saúde Pública, Rio de Janeiro, v. 25, n. 4, p. 898-906, abr. 2009.

FRANCO, Túlio B.; MERHY, Emerson E. PSF: contradições e novos desafios. Campinas, mar. 1999. Disponível em: <www.uff.br/ saudecoletiva/professores/merhy/artigos-17. pdf $>$. Acesso em: $1^{\circ}$ ago. 2014.

GALAVOTE, Heletícia et al. Desvendando os processos de trabalho do agente comunitário de saúde nos cenários revelados na Estratégia Saúde da Família no município de Vitória (ES, Brasil). Ciência \& Saúde Coletiva, Rio de Janeiro, v. 16, n. 1, p. 231-240, jan. 2011.

GARCIA, Ana C. et al. Perfil dos trabalhadores na Estratégia Saúde da Família do Estado Espírito Santo. Relatório técnico. Vitória: Fapes, 2014. (Impresso).

GIOVANELLA, Lígia; ESCOREL, Sarah; MENDONÇA, Maria H. (coord.). Estudos de caso sobre implementação da Estratégia Saúde da Família em quatro grandes centros urbanos: Vitória. Relatório final. Brasília: Ministério da Saúde, 2009. Disponível em: <www5.ensp.fiocruz.br/biblioteca/home/ exibedetalhesBiblioteca.cfm $? \mathrm{ID}=9436 \&$ tipo $=\mathrm{B}>$. Acesso em 18 out. 2016.

GRANDO, Maristel K.; DALL'AGNOL, Clarice, M. Desafios do processo grupal em reuniões de equipe da Estratégia Saúde da Família. Escola Anna Nery Revista de Enfermagem, Rio de Janeiro, v. 14, n. 3, p. 504-510, 2010.

INSTITUTO BRASILEIRO DE GEOGRAFIA E ESTATÍSTICA (IBGE). Censo Demográfico 2010. Disponível em: <http://censo2010.ibge. gov.br>. Acesso em: 18 out. 2016.

JARDIM, Tatiana A.; LANCMAN, Selma. Aspectos subjetivos do morar e trabalhar na mesma comunidade: a realidade vivenciada pelo agente comunitário de saúde. Interface: Comunicação, Saúde e Educação, Botucatu, v. 13, n. 28, p. 123-135, 2009.

LUNARDELO, Simone R. O trabalho do agente comunitário de saúde nos núcleos de saúde da família em Ribeirão Preto. 154 f. Dissertação (Mestrado em Enfermagem) - Escola de Enfermagem 
de Ribeirão Preto, Universidade de São Paulo, Ribeirão Preto, 2004.

MARZARI, Carla K.; JUNGES, José R.; SELLI, Lucilda. Agentes comunitários de saúde: perfil e formação. Ciência \& Saúde Coletiva, Rio de Janeiro, v. 16, supl. 1, p. 873-880, 2011.

MENEGUSSI, Juliana M.; OGATA, Márcia N.; ROSALINI, Maria H. P. O agente comunitário de saúde como morador, trabalhador e usuário em São Carlos, São Paulo. Trabalho, Educação e Saúde, Rio de Janeiro, v. 12, n. 1, p. 87-106, jan.-abr. 2014.

MERHY, Emerson E. Saúde: a cartografia do trabalho vivo em ato. 3. ed. São Paulo: Hucitec, 2002.

MONKEN, Maurício; BARCELLOS, Christovam. O território na promoção e vigilância em saúde. In: FONSECA, Angélica F. (org.). O território e o processo saúde-doença. Rio de Janeiro: EPSJV/Fiocruz, 2007. Disponível em: <www. epsjv.fiocruz.br/pdtsp/index.php?livro_id= 6\&area_id $=2$ \&autor_id $=$ \&capitulo_id $=22$ \&arquivo=ver_conteudo_2 $>$. Acesso em: 18 out. 2016.

MOTA, Roberta R. A.; DAVID, Helena M. S. L. A crescente escolarização do agente comunitário de saúde: uma indução do processo de trabalho? Trabalho, Educação e Saúde, Rio de Janeiro, v. 8, n. 2, p. 229-248, jul.-out. 2010.

NOGUEIRA, Roberto P.; SILVA, Frederico B.; RAMOS, Zuleide V. O. A vinculação institucional de um trabalhador sui generis: o agente comunitário de saúde. Rio de Janeiro: Ipea, jun. 2000. (Textos para Discussão, 735).
SANTOS, Karina T. et al. Agente comunitário de saúde: perfil adequado a realidade do Programa Saúde da Família? Ciência \& Saúde Coletiva, Rio de Janeiro, v. 16, supl. 1, p. 1.023-1.028, 2011.

SILVA, José P. V.; BATISTELLA, Carlos; GOMES, Mauro L. Problemas, necessidades e situação de saúde: uma revisão de abordagens para reflexão e ação da equipe de saúde da família. In: FONSECA, Angélica F. (org.). $O$ território e o processo saúde-doença. Rio de Janeiro: EPSJV/Fiocruz, 2007. p. 159-176.

SILVA, Maria J.; RODRIGUES, Rui M. O agente comunitário de saúde no processo de municipalização da saúde. Revista Eletrônica de Enfermagem, Goiânia, v. 2, n. 1, jan.-jun. 2000. Disponível em: <https://revistas.ufg. br/fen/article/view/678/748>. Acesso em: 15 jul. 2014.

URSINE, Bárbara L.; TRELHA, Celita S.; NUNES, Elisabete F. P. A. O agente comunitário de saúde na Estratégia de Saúde da Família: uma investigação das condições de trabalho e da qualidade de vida. Revista Brasileira de Saúde Ocupacional, São Paulo, v. 35, n. 122, jul./dez. 2010. Disponível em: $<$ www.scielo.br/scielo.php?script $=$ sci arttext\&pid $=$ S0303-76572010000200015>. Acesso em 18 out. 2016.

Recebido em 22/10/2014

Aprovado em 18/11/2015 